\title{
Distribuição da Gordura Corporal e Perfis Lipídico e Glicêmico de Pacientes Infectados Pelo HIV
}

\section{artigo original}

\author{
Milena Maria M. Guimarães \\ DiRCEU BARTOLOMEU GRECO \\ ANTÔNIO RIBEIRO DE O. JÚNIOR \\ Mariana Guimarães Penido \\ LUCAS JOSÉ DE C. MACHADO
}

Serviço Especial de Endocrinologia e Metabologia (AROJ \& LJCM) e Serviço de Doenças Infecciosas e Parasitárias do Hospital das Clínicas da UFMG (DBG), e Departamento de Clínica Médica da Faculdade de Medicina da UFMG (MMMG \& MGP), Belo Horizonte, MG.

Recebido em 16/02/06

Revisado em 28/06/06 Aceito em 04/08/06

\author{
RESUMO
}

Os objetivos foram avaliar dados antropométricos e perfis lipídico e glicêmico de pacientes infectados pelo HIV usuários e não usuários de anti-retrovirais (ARV), e verificar a associação entre ARV e alterações da gordura corporal, distúrbios lipídicos e da homeostase da glicose. Foram incluídos 176 pacientes (133 usuários e 43 não usuários de ARV). Os pacientes foram submetidos a avaliação clínica, exames laboratoriais, ultrassonografia, biompedanciometria e medida de pregas cutâneas. Pacientes usuários de ARV apresentaram maior relação cintura/quadril ( $p=$ $0,0002)$, maior espessura da gordura intra-abdominal medida pela ultrassonografia $(p=0,003)$ e menores pregas de gordura bicipital $(p=0,01)$ e tricipital $(p=0,0002)$. Estes pacientes tiveram níveis mais elevados de triglicérides $(p=0,0002)$, colesterol total $(p=0,00007)$ e colesterol HDL $(p=$ 0,009 ). Eles também apresentaram maiores níveis de glicose aos 60 ( $p=$ $0,01)$ e 120 minutos $(p=0,001)$ após dextrosol, maiores níveis de insulina de jejum $(p=0,03)$ e maiores valores do índice $\operatorname{HOMA}(p=0,02)$. As drogas anti-retrovirais estão associadas a acúmulo central e perda periférica de gordura. Além disso, estas drogas estão associadas a alterações lipídicas e a aumento da resistência insulínica, conhecidos fatores de risco cardiovascular. (Arq Bras Endocrinol Metab 2007;51/1:42-51)

Descritores: HIV; Terapia anti-retroviral; Efeitos adversos; Síndrome lipodistrófica do HIV; Lipodistrofia; Síndrome metabólica do HIV

\section{ABSTRACT}

Corporal Fat Distribution and Lipidic and Glicemic Profiles of HIVInfected Patients.

The aims of this study were to describe anthropometric data and glycemic and lipidic profiles of HIV-infected patients treated or not with antiretrovirals (ARV) drugs, and to assess association between these drugs and body composition changes, lipid and glucose homeostasis disturbances. There were 176 patients included (133 ARV-treated patients and 43 ARV-naïve). The patients were submitted to clinical evaluation, laboratorial analysis, ultrasonographic measurements, bioelectrical impedance analysis and skin folds thickness measurements. The ARV-treated group showed higher waist-tohip ratio ( $p=0.0002)$, higher intra-abdominal fat thickness measured by ultrasonography $(p=0.003)$ and lower bicipital $(p=0.01)$ and tricipital $(p=0.0002)$ skin folds. This group also showed higher triglyceride $(p=0.0002)$, total cholesterol ( $p=0.00007)$, HDL cholesterol $(p=0.009)$, glucose measurements one hour $(p=0.01)$ and two hours $(p=0.001)$ after dextrose load, higher levels of fasting insulin $(p=0.03)$ and higher HOMAR index $(p=0.02)$. The antiretroviral drugs are associated with increased visceral fat and decreased peripheral fat pads. Beside that, these drugs are associated with atherogenic lipid profile and insulin resistance, two independent risk predictors of cardiovascular disease. (Arq Bras Endocrinol Metab 2007;51/1:42-51)

Keywords: HIV; Antiretroviral therapy; Side-effect; Lipodystrophy syndrome HIV; Lipodystrophy; Metabolic syndrome HIV 
A

PARTIR DE 1996, QUANDO a terapia anti-retroviral de alta potência (highly active antiretroviral thera$p y$, ou simplesmente HAART) tornou-se uma rotina no tratamento de pacientes infectados pelo HIV, mudanças na distribuição da gordura corporal e alterações do perfil lipídico e glicêmico foram observadas (1-4). Estas alterações foram posteriormente denominadas de síndrome lipodistrófica do HIV, que pode ser melhor definida como perda dos depósitos de gordura periférica (lipoatrofia) e/ou acúmulo de gordura central (lipo-hipertrofia) e/ou manifestações metabólicas lipídicas e glicêmicas (5).

É bem conhecido que a distribuição regional da gordura, em particular o tecido gorduroso intra-abdominal, está associado com desordens metabólicas neuroendócrinas, principalmente resistência insulínica e síndrome metabólica, e com um grande aumento da morbidade e da mortalidade cardiovascular (6). Estes riscos são observados até mesmo em indivíduos não obesos, se estes apresentam um aumento de depósitos de gordura visceral (6). Portanto, parece razoável supor que em função da tendência a acúmulo de gordura visceral, os pacientes infectados pelo HIV em uso das drogas anti-retrovirais (ARV) apresentem maior risco para doenças ateroscleróticas.

A melhora considerável na condução destes pacientes, notadamente o uso da terapia anti-retroviral de alta potência, tem progressivamente transformando a infecção pelo HIV em uma doença crônica (7-9). Considerando o aumento da expectativa de vida destes pacientes, é muito importante propor uma avaliação sistemática e precoce dos riscos para eventos cardiovasculares nesta população.

O objetivo deste estudo foi descrever dados antropométricos e os perfis lipídico e glicêmico de pacientes infectados pelo HIV tratados ou não com drogas anti-retrovirais, e verificar a associação entre estas drogas e as alterações da distribuição da gordura corporal, alterações lipídicas e distúrbios da homeostase da glicose.

\section{PACIENTES E MÉTODOS}

Trata-se de estudo transversal observacional. Os pacientes, todos acima de 18 anos, foram recrutados no Serviço de Doenças Infecciosas e Parasitárias do Hospital das Clínicas da Universidade Federal de Minas Gerais (Centro de Treinamento e Referência em Doenças Infecciosas e Parasitárias Orestes Diniz, fruto de convênio entre a Universidade e a Prefeitura Municipal de Belo Horizonte). Foram incluídos no estudo pacientes que estivessem recebendo ARV por pelo menos três meses ou que não tivessem nunca feito uso de
ARV. O protocolo da pesquisa foi aprovado pelo Comitê de Ética em Pesquisa da Universidade Federal de Minas Gerais (UFMG) e foi obtido termo de consentimento livre e esclarecido de todos os pacientes.

Foram excluídos do estudo pacientes com alterações metabólicas (por exemplo, hiperlipidemia, diabetes mellitus) ou lipodistrofia antes do diagnóstico de infecção por HIV; pacientes em uso de glicocorticóides ou outros esteróides, hormônio do crescimento, beta-bloqueadores e/ou tiazídi$\cos$, ou outros fármacos que induzam alterações metabólicas e/ou de distribuição da gordura corporal; portadores de afecção clinicamente relevante que pudesse dificultar a implementação ou interpretação dos resultados do estudo; grávidas ou lactantes; portadores de alguma condição mental que impedisse a compreensão da natureza, escopo e possíveis conseqüências do estudo.

Os pacientes foram submetidos a avaliação clínica, exames laboratoriais, ultrassonografia, biompedanciometria e medida de pregas cutâneas. Todos os dados antropométricos foram realizados pelo mesmo examinador.

A medida da cintura foi definida como o menor diâmetro entre a última costela e a crista ilíaca, estando o indivíduo de pé, em expiração (10) e a medida do quadril foi definida como a maior largura na região dos grandes trocânteres (10). A relação cintura/quadril também foi calculada.

As pregas cutâneas foram medidas com adipômetro Lange ${ }^{\circledR}$ e as medidas foram feitas em triplicata, sempre do lado direito do corpo, diretamente na pele, nas seguintes localizações: tríceps, bíceps, subescapular e supra-ilíaca. O percentual de gordura corporal foi calculado utilizando-se as quatro pregas cutâneas medidas (11).

O percentual de gordura corporal também foi avaliado através de impedanciometria bioelétrica tetrapolar, utilizando-se Monitor de Composição Corporal por Bioimpedância Biodynamics ${ }^{\circledR}$, modelo 310e (12).

Os pacientes foram submetidos a ultrassonografia (US) de abdome para avaliação da espessura da gordura intra-abdominal e subcutânea. $\mathrm{O}$ exame foi realizado $\mathrm{em}$ aparelho ultrassonográfico Siemens ${ }^{\circledR}$, modelo Sonoline Prima, com transdutor convexo eletrônico de 3,5 MHz, por um mesmo examinador que não tinha maiores conhecimentos a respeito do uso de anti-retrovirais pelos pacientes. $\mathrm{O}$ paciente, após jejum de seis horas, era colocado em decúbito dorsal. As medidas foram realizadas em triplicata, em região imediatamente superior à cicatriz umbilical, na linha xifoumbilical. Considerou-se espessura subcutânea a medida entre pele e face externa do músculo reto abdominal, e espessura intra-abdominal a medida entre face interna do músculo reto abdominal e a parede posterior da aorta $(10,13)$. A reprodutibilidade intra-examinador destas medidas foi avaliada através de tripla determinação em dez indivíduos em dois diferentes dias.

Amostras sanguíneas foram obtidas de cada paciente após 12 horas de jejum e uma e duas horas após sobrecarga com $75 \mathrm{~g}$ de dextrosol. Glicose, colesterol total e triglicérides foram determinados enzimaticamente. Colesterol HDL foi determinado por imunoinibição e a insulina foi dosada através 
de quimioluminescência. Resistência insulínica foi estimada pelo Homeostasis Model Assessment Insulin Resistance (HOMA-IR), utilizando-se a seguinte equação: insulina em jejum $(\mu \mathrm{U} / \mathrm{mL})$ x glicose em jejum $(\mathrm{mmol} / \mathrm{L}) / 22,5$ (14).

Diferenças entre os grupos foram avaliadas usando-se análise de variância seguida pelo teste $\mathrm{t}$ de Student ou pelo teste de Kruskal-Wallis. O nível de significância foi de $p<0,05$. A reprodutibilidade intra-examinador foi avaliada pelo teste $t$ de Student e pela variação dos coeficientes de diferenças.

\section{RESULTADOS}

Foram incluídos no estudo 176 pacientes, sendo 133 (76\%) usuários de drogas anti-retrovirais e $43(24 \%)$ não usuários. A idade da população total variou de 20 a 62 anos, com média de 39,5 $\pm 9,0$ anos. A maioria dos pacientes avaliados era do sexo masculino (64\%) e tinha escolaridade superior a oito anos completos $(65 \%)$. Predominaram os solteiros $(61 \%)$ e a via sexual foi a principal responsável pela transmissão do vírus $(89 \%)$. Não houve diferença estatística entre os grupos em relação a sexo, escolaridade, estado civil e provável forma de infecção.

Hipertensão arterial sistêmica (HAS), tabagismo e sedentarismo foram observados em $8 \%, 32 \%$ e $80 \%$ dos pacientes, respectivamente. Os dois grupos avaliados, usuários ou não de drogas anti-retrovirais, mostraram-se estatisticamente similares em relação a tabagismo, presença de HAS e sedentarismo, assim como em relação à história familiar de diabetes mellitus, de acidente vascular cerebral, de doença coronariana, de dislipidemia e de HAS.

A tabela 1 mostra de forma esquemática quantos dos 133 pacientes estavam usando drogas das três principais classes de anti-retrovirais. Praticamente todos os pacientes (131) usavam inibidores da transcriptase reversa análogos de nucleosídeos (ITRN).

$\mathrm{Na}$ tabela 2 são resumidos os dados coletados relacionados ao perfil imunológico dos pacientes, tendo sido observada diferença estatisticamente significativa entre os dois grupos apenas em relação à viremia plasmática $(p=0,000002$, pelo teste $t$ de Student).

Tabela 1. Número de pacientes em uso das três classes de drogas anti-retrovirais.

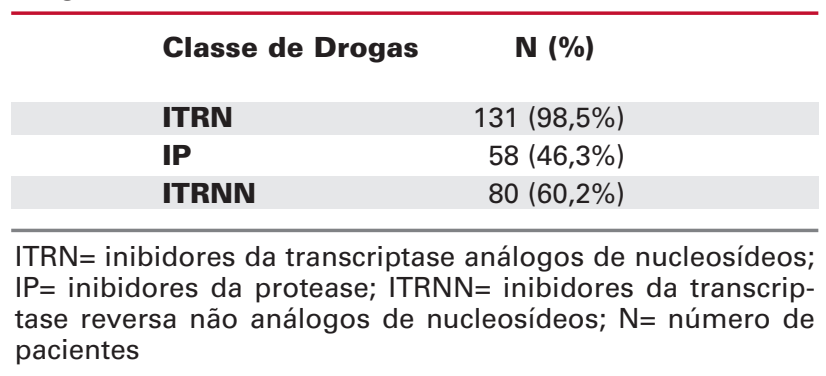

Tabela 2. Descrição do perfil imunológico e comparação entre os grupos.

\begin{tabular}{|c|c|c|c|c|}
\hline Variável & Média (DP) & Mediana & Teste Estatístico & Valor p \\
\hline Carga viral (cópias de RNA/ml) & & & Kruskal-Wallis & 0,000002 \\
\hline População total $(n=148)$ & $26.573,5(92.558,0)$ & 400,0 & & \\
\hline Pacientes com ARV $(n=114)$ & $18.291,0(70.275,0)$ & 80,0 & & \\
\hline Pacientes sem ARV $(n=34)$ & $54.345,0(142.195,0)$ & $12.311,0$ & & \\
\hline Contagem de linfócitos CD4 + (cels/ml) & & & t de Student & 0,20 \\
\hline População total $(n=158)$ & $419,0(239,5)$ & 386,0 & & \\
\hline Pacientes com ARV ( $n=119)$ & $405,0(223,9)$ & 385,0 & & \\
\hline Pacientes sem ARV $(n=39)$ & $462,1(280,5)$ & 433,0 & & \\
\hline Relação CD4/CD8 & & & t de Student & 0,11 \\
\hline População total $(n=153)$ & $0,48(0,31)$ & 0,42 & & \\
\hline Pacientes com ARV $(n=115)$ & $0,46(0,29)$ & 0,40 & & \\
\hline Pacientes sem ARV $(n=38)$ & $0,55(0,37)$ & 0,50 & & \\
\hline
\end{tabular}

$\mathrm{DP}=$ desvio-padrão; $\mathrm{ARV}=$ drogas anti-retrovirais; $\mathrm{p}=$ probabilidade de significância 
A tabela 3 mostra os valores de peso, IMC, cintura, quadril e relação cintura/quadril para todos os pacientes. Houve diferença estatística entre os grupos somente para a variável relação cintura/quadril, que foi maior entre os usuários de ARV. Quando avaliadas as populações feminina e masculina, separadamente, observou-se também que apenas a relação cintura/ quadril foi estatisticamente diferente entre os grupos, sendo também maior nos pacientes em uso de ARV ( $\operatorname{com} p=0,01$ na população feminina e $p=0,05$ na população masculina, pelo teste $t$ de Student).

A tabela 4 apresenta as medidas de paquimetria. Pacientes usuários de ARV apresentaram espessuras das pregas de bíceps e tríceps menores que os não usuários. Quando avaliadas as populações feminina e masculina separadamente, apenas entre os homens se observou uma menor espessura das pregas cutâneas de bíceps e tríceps $(p=0,03$ e 0,01 respectivamente, pelo teste de Kruskal-Wallis).

Em relação à impedanciometria, não houve diferença estatística entre os grupos para nenhuma das variáveis, inclusive quando avaliadas as populações feminina e masculina separadamente.

Dos 176 pacientes submetidos à avaliação clínica, 160 (91\%) compareceram para a realização da ultrassonografia, sendo 123 pacientes em uso de ARV e 37 pacientes não usuários. O teste de reprodutibilidade intra-examinador das medidas ultrassonográficas demonstrou variação de 4,6\% e 2,5\% para gordura subcutânea e intra-abdominal, respectivamente.
A tabela 5 mostra as medidas da gordura subcutânea e intra-abdominal feitas pela ultrassonografia. Pacientes usuários de ARV apresentaram espessura de gordura intra-abdominal maior que não usuários. Todavia, quando se analisou a população feminina e masculina separadamente, não se observou diferença estatisticamente representativa entre os grupos.

Dos 176 pacientes submetidos à avaliação clínica, $172(98 \%)$ compareceram ao Laboratório Central do Hospital das Clínicas para coleta de amostras sanguíneas, sendo 130 pacientes em uso de ARV e 42 pacientes não usuários.

A tabela 6 apresenta a descrição do perfil lipídico da população estudada e dos grupos em questão. Os níveis plasmáticos de triglicérides, colesterol total, colesterol VLDL e colesterol HDL nos pacientes em uso de ARV foram significativamente maiores que os observados nos não usuários.

A tabela 7 apresenta os valores de glicemia de jejum e após sobrecarga com dextrosol e os valores da área sob a curva da variação glicêmica para a população total estudada. Os pacientes em uso de ARV apresentaram resposta glicêmica maior que os não usuários.

A tabela 8 mostra os valores da insulina de jejum, aos 60 e 120 minutos após sobrecarga com dextrose, e a curva da variação de insulina para a população total. Os pacientes usuários de ARV apresentaram insulinemia basal maior que os não usuários.

Tabela 3. Dados antropométricos.

\begin{tabular}{|c|c|c|c|c|}
\hline Variável & Média (DP) & Mediana & Teste Estatístico & Valor $\mathbf{p}$ \\
\hline Peso (kg) & & & Kruskal-Wallis & 0,60 \\
\hline População total & $65,6(13,1)$ & 63,6 & & \\
\hline Pacientes com ARV & $65,6(12,0)$ & 64,0 & & \\
\hline Pacientes sem ARV & $65,7(16,3)$ & 62,4 & & \\
\hline $\mathrm{IMC}\left(\mathrm{kg} / \mathrm{m}^{2}\right)$ & & & Kruskal-Wallis & 0,50 \\
\hline População total & $23,8(4,2)$ & 23,1 & & \\
\hline Pacientes com ARV & $23,8(3,8)$ & 23,1 & & \\
\hline Pacientes sem ARV & $23,7(5,1)$ & 23,1 & & \\
\hline Cintura $(\mathrm{cm})$ & & & Kruskal-Wallis & 0,14 \\
\hline População total & $84,9(10,5)$ & 84,0 & & \\
\hline Pacientes com ARV & $85,5(9,4)$ & 85,0 & & \\
\hline Pacientes sem ARV & $83,3(13,1)$ & 82,0 & & \\
\hline Quadril (cm) & & & Kruskal-Wallis & 0,38 \\
\hline População total & $91,2(8,9)$ & 89,3 & & \\
\hline Pacientes com ARV & $90,7(8,2)$ & 89,0 & & \\
\hline Pacientes sem ARV & $92,8(10,7)$ & 90,0 & & \\
\hline Relação cintura/quadril & & & t de Student & 0,0002 \\
\hline População total & $0,93(0,07)$ & 0,94 & & \\
\hline Pacientes com ARV & $0,94(0,07)$ & 0,95 & & \\
\hline Pacientes sem ARV & $0,90(0,07)$ & 0,88 & & \\
\hline
\end{tabular}

$\mathrm{DP}=$ desvio-padrão; $\mathrm{ARV}=$ drogas anti-retrovirais; $\mathrm{p}=$ probabilidade de significância

$\mathrm{n}=176$ na população total; $\mathrm{n}=133$ no grupo usuário de $\mathrm{ARV} ; \mathrm{n}=43$ no grupo não usuário de ARV 
Tabela 4. Espessura das pregas cutâneas e percentual de gordura corporal.

\begin{tabular}{|c|c|c|c|c|}
\hline Variável & Média (DP) & Mediana & Teste Estatístico & Valor $\mathbf{p}$ \\
\hline Bíceps (mm) & & & Kruskal-Wallis & 0,01 \\
\hline População total & $6,9(4,4)$ & 5,2 & & \\
\hline Pacientes com ARV & $6,5(4,0)$ & 5,0 & & \\
\hline Pacientes sem ARV & $8,3(5,4)$ & 7,0 & & \\
\hline Tríceps (mm) & & & Kruskal-Wallis & 0,0002 \\
\hline População total & $9,9(6,0)$ & 8,0 & & \\
\hline Pacientes com ARV & $9,0(5,2)$ & 7,3 & & \\
\hline Pacientes sem ARV & $12,7(7,2)$ & 10,7 & & \\
\hline Suprailíaca (mm) & & & t de Student & 0,9 \\
\hline População total & $12,2(6,7)$ & 11,0 & & \\
\hline Pacientes com ARV & $12,2(6,5)$ & 11,0 & & \\
\hline Pacientes sem ARV & $12,3(7,3)$ & 12,0 & & \\
\hline Subescapular (mm) & & & t de Student & 0,76 \\
\hline População total & $20,0(9,2)$ & 17,7 & & \\
\hline Pacientes com ARV & $20,1(9,0)$ & 17,6 & & \\
\hline Pacientes sem ARV & $19,6(9,9)$ & 18,0 & & \\
\hline$\%$ de gordura (através das pregas) & & & t de Student & 0,2 \\
\hline População total & $23,7(7,8)$ & 23,1 & & \\
\hline Pacientes com ARV & $23,3(7,6)$ & 22,9 & & \\
\hline Pacientes sem ARV & $25,1(8,6)$ & 24,6 & & \\
\hline
\end{tabular}

$\mathrm{DP}=$ desvio-padrão; $\mathrm{ARV}=$ drogas anti-retrovirais; $\mathrm{p}=$ probabilidade de significância

$n=176$ na população masculina; $n=133$ no grupo usuário de $A R V ; n=43$ no grupo não usuário de $A R V$

Tabela 5. Dados da ultrassonografia.

\begin{tabular}{|c|c|c|c|c|}
\hline Variável & Média (DP) & Mediana & Teste Estatístico & Valor $\mathbf{p}$ \\
\hline Espessura da gordura subcutânea $(\mathrm{mm})$ & & & Kruskal-Wallis & 0,24 \\
\hline População total & $18,9(10,5)$ & 15,6 & & \\
\hline Pacientes com ARV & $18,1(9,5)$ & 15,5 & & \\
\hline Pacientes sem ARV & $21,8(13,1)$ & 17,4 & & \\
\hline Espessura da gordura intra-abdominal (mm) & & & t de Student & 0,03 \\
\hline População total & $66,9(20,6)$ & 64,5 & & \\
\hline Pacientes com ARV & $68,9(21,1)$ & 66,8 & & \\
\hline Pacientes sem ARV & $60,4(17,7)$ & 57,2 & & \\
\hline
\end{tabular}

$\mathrm{DP}=$ desvio-padrão; $\mathrm{ARV}=$ drogas anti-retrovirais; $\mathrm{p}=$ probabilidade de significância $n=160$ na população total; $n=123$ no grupo com ARV; $n=37$ no grupo sem ARV

Houve diferença estatística entre os grupos também quando calculado o índice HOMA-IR, sendo $2,1 \pm 3,0$ nos pacientes usuários de ARV e $1,3 \pm 1,0$ nos pacientes sem ARV, com $p=0,02$ pelo teste de Kruskal-Wallis.

\section{DISCUSSÃo}

A maior parte dos estudos transversais avaliou pacientes em uso de ARV comparando pacientes com ou sem lipodistrofia ou com ou sem uso de inibidores de protease. Outros estudos compararam pacientes infectados em uso de ARV com indivíduos sadios. Neste estudo, optamos por comparar pacientes com e sem exposição a ARV para avaliar o impacto do uso combinado de anti-retrovirais nas medidas de distribuição de gordura e nos perfis lipídico e glicêmico dos pacientes em nosso meio.

A freqüência de utilização por classe de drogas em nosso estudo [98,5\% em uso de inibidores da transcriptase reversa análogos de nucleosídeos (ITRN), 46\% usavam inibidores de protease (IP) e $60 \%$ usavam inibidores da transcriptase reversa não análogos de 
Tabela 6. Perfil lipídico da população.

\begin{tabular}{|c|c|c|c|c|}
\hline Variável & Média (DP) & Mediana & Teste Estatístico & Valor p \\
\hline Triglicérides (mg/dl) & & & Kruskal-Wallis & 0,00002 \\
\hline População total & $194,8(180,8)$ & 135,5 & & \\
\hline Pacientes com ARV & $221,2(199,2)$ & 150,5 & & \\
\hline Pacientes sem ARV & $113,2(50,2)$ & 99,5 & & \\
\hline Colesterol total (mg/dl) & & & Kruskal-Wallis & 0,00007 \\
\hline População total & $185,3(59,0)$ & 177,0 & & \\
\hline Pacientes com ARV & $194,6(61,1)$ & 187,5 & & \\
\hline Pacientes sem ARV & $156,6(40,3)$ & 152,0 & & \\
\hline Colesterol HDL (mg/dl) & & & $\mathrm{t}$ de Student & 0,009 \\
\hline População total & $34,4(12,5)$ & 34,0 & & \\
\hline Pacientes com ARV & $35,8(12,5)$ & 35,0 & & \\
\hline Pacientes sem ARV & $30,1(11,5)$ & 28,5 & & \\
\hline Colesterol VLDL (mg/dl) & & & Kruskal-Wallis & 0,001 \\
\hline População total & $29,5(15,4)$ & 25,0 & & \\
\hline Pacientes com ARV & $31,8(16,3)$ & 27,0 & & \\
\hline Pacientes sem ARV & $22,6(10,0)$ & 20,0 & & \\
\hline Colesterol LDL (mg/dl) & & & t de Student & 0,057 \\
\hline População total & $113,5(38,8)$ & 108,0 & & \\
\hline Pacientes com ARV & $117,1(39,9)$ & 112,0 & & \\
\hline Pacientes sem ARV & $103,8(34,2)$ & 94,0 & & \\
\hline
\end{tabular}

$\mathrm{DP}=$ desvio-padrão; $\mathrm{ARV}=$ drogas anti-retrovirais; $\mathrm{p}=$ probabilidade de significância

$\mathrm{n}=172$ na população total; $\mathrm{n}=130$ no grupo dos usuários; $\mathrm{n}=42$ no grupo dos não usuários

Tabela 7. Perfil glicêmico e área sob a curva glicêmica da população.

\begin{tabular}{|c|c|c|c|c|}
\hline Variável & Média (DP) & Mediana & Teste Estatístico & Valor $p$ \\
\hline Glicemia de jejum (mg/dl) & & & Kruskal-Wallis & 0,15 \\
\hline População total & $88(17,6)$ & 86 & & \\
\hline Pacientes com ARV & $89(19,4)$ & 87 & & \\
\hline Pacientes sem ARV & $84(8,9)$ & 85 & & \\
\hline Glicemia 60 minutos pós-dextrose (mg/dl) & & & Kruskal-Wallis & 0,01 \\
\hline População total & $131(52,9)$ & 124 & & \\
\hline Pacientes com ARV & $137(56,0)$ & 130 & & \\
\hline Pacientes sem ARV & $114(37,1)$ & 108 & & \\
\hline Glicemia 120 minutos pós-dextrose (mg/dl) & & & Kruskal-Wallis & 0,001 \\
\hline População total & $114(50,0)$ & 106 & & \\
\hline Pacientes com ARV & $120(54,0)$ & 110 & & \\
\hline Pacientes sem ARV & $95(27,7)$ & 93 & & \\
\hline Área sob a curva glicêmica (mg/dl/120 min) & & & Kruskal-Wallis & 0,009 \\
\hline População total & $3381(3500)$ & 3045 & & \\
\hline Pacientes com ARV & 3791 (3697) & 3315 & & \\
\hline Pacientes sem ARV & $2114(2431)$ & 1515 & & \\
\hline
\end{tabular}

$\mathrm{DP}=$ desvio-padrão; $\mathrm{PD}=$ pós-dextrosol; $\mathrm{ARV}=$ drogas anti-retrovirais; $\mathrm{p}=$ probabilidade de significância $n=172$ na população total; $n=130$ no grupo dos usuários; $n=42$ no grupo dos não usuários 
Tabela 8. Perfil insulinêmico e área sob a curva insulinêmica da população.

\begin{tabular}{|c|c|c|c|c|}
\hline Variável & Média (DP) & Mediana & Teste Estatístico & Valor $\mathbf{p}$ \\
\hline Insulina de jejum $(\mu \mathrm{U} / \mathrm{ml})$ & & & Kruskal-Wallis & 0,03 \\
\hline População total & $8,1(8,1)$ & 6,4 & & \\
\hline Pacientes com ARV & $8,9(9,0)$ & 6,7 & & \\
\hline Pacientes sem ARV & $6,1(3,8)$ & 5,4 & & \\
\hline Insulina 60 minutos pós-dextrose (mg/dl) & & & $t$ de Student & 0,58 \\
\hline População total & $81,0(95,9)$ & 46,8 & & \\
\hline Pacientes com ARV & $81,7(87,8)$ & 51,7 & & \\
\hline Pacientes sem ARV & $72,5(108,8)$ & 40,8 & & \\
\hline Insulina 120 minutos pós-dextrose (mg/dl) & & & $t$ de Student & 0,14 \\
\hline População total & $55,2(57,7)$ & 34,8 & & \\
\hline Pacientes com ARV & $59,2(57,6)$ & 38,9 & & \\
\hline Pacientes sem ARV & $44,2(54,8)$ & 25,7 & & \\
\hline Área sob a curva insulinêmica $(\mathrm{mg} / \mathrm{dl} / 120 \mathrm{~min})$ & & & Kruskal-Wallis & 0,051 \\
\hline População total & $6756(8141)$ & 4095 & & \\
\hline Pacientes com ARV & 7207 (8575) & 4560 & & \\
\hline Pacientes sem ARV & $5000(5050)$ & 3375 & & \\
\hline
\end{tabular}

$\mathrm{DP}=$ desvio-padrão; $\mathrm{PD}=$ pós-dextrosol; $\mathrm{ARV}=$ drogas anti-retrovirais; $\mathrm{p}=$ probabilidade de significância $\mathrm{n}=172$ na população total; $\mathrm{n}=130$ no grupo dos usuários; $\mathrm{n}=42$ no grupo dos não usuários

nucleosídeos (ITRNN)] reflete as atuais recomendações para terapia anti-retroviral em adultos e adolescentes infectados pelo HIV, elaboradas pela coordenação nacional de DST/AIDS (15). A terapia inicial recomendada é composta por dois ITRN associada a um ITRNN ou a um IP. Mudanças no esquema inicial podem ser necessárias devido a falha terapêutica, toxicidade ou intolerância ao tratamento. Estudos prévios demonstravam um maior número de pacientes em uso de IP, como o estudo canadense que avaliou via questionário um total de 1.035 pacientes, sendo que $62 \%$ destes usavam esquema com drogas IP e $38 \%$ usavam esquema com ITRNN (16). Devido à sua boa tolerabilidade e sua boa potência anti-retroviral, as drogas ITRNN estão sendo utilizadas cada vez mais freqüentemente. O desenho transversal e o tamanho da nossa amostra limitaram a avaliação do impacto nas alterações estudadas de cada uma das classes separadamente.

Em nosso estudo, o uso de ARV não parece estar associado a alteração na gordura corporal global, pois peso, IMC e porcentagem de gordura corporal pela biompedanciometria ou paquimetria foram semelhantes entre os pacientes usuários ou não de antiretrovirais. Entretanto, os pacientes usuários de ARV tiveram maiores relação cintura/quadril e espessura da gordura visceral medida pelo ultra-som, e menores pregas biceptal e triceptal, demonstrando uma modificação da distribuição de gordura, com acúmulo de gordura central e perda de gordura periférica.

A maioria dos estudos também demonstrou não haver diferença estatisticamente significativa quanto a peso e IMC quando comparados pacientes em uso de ARV com indivíduos não infectados pelo HIV (17-19) ou a grupo virgem de tratamento (20-22).

Ao contrário dos nossos resultados, Saint-Marc e cols. (22) observaram não haver diferença estatística entre os pacientes usuários de ARV e os não usuários no que diz respeito à relação cintura/quadril; entretanto, observaram uma menor espessura das pregas no bíceps, tríceps e supra-ilíaca entre os pacientes infectados em uso de ARV, corroborando a hipótese de perda subcutânea de gordura associada a estas drogas.

Cabe ressaltar que a medida da cintura tem sido proposta como melhor indicador de risco que a relação cintura/quadril. Segundo Deprés e cols. (23), uma cintura superior a 0,9 e 1,0 metro para mulheres e homens respectivamente, indica um acúmulo de gordura visceral medido por tomografia computadorizada de pelo menos $130 \mathrm{~cm}^{2}$, valor este relacionado a várias anormalidades metabólicas. Vários são os estudos que demonstram uma melhor correlação da medida da cintura com a obesidade central e o aumento de risco cardiovascular quando comparada à relação cintura/ quadril $(24,25)$. Contudo, o nosso estudo não mostrou diferença estatística entre os grupos estudados (usuários e não usuários de $\mathrm{ARV}$ ) em relação à medida da cintura.

Por se tratar de método de fácil acesso, de baixo custo, com efeitos adversos mínimos para o paciente e por já ter sido validada na população brasileira (10), optamos pela ultrassonografia para medida da espessura da gordura intra-abdominal. Vários trabalhos têm 
demonstrado boa correlação entre as medidas da gordura intra-abdominal feita pela ultrassonografia e pela tomografia computadorizada, considerada o método padrão-ouro para a quantificação de gordura intraabdominal $(10,13)$.

Martínez e cols. (26) realizaram um estudo avaliando 60 pacientes infectados pelo HIV em uso de ARV e 60 pacientes não infectados. Utilizando ultrassonografia, eles mediram a espessura da gordura subcutânea na região peri-umbilical, braquial e malar e a espessura da gordura intra-abdominal. Eles observaram uma maior gordura intra-abdominal e uma menor gordura subcutânea braquial e malar nos pacientes com lipodistrofia definida clinicamente quando comparados aos sem lipodistrofia. Não foram incluídos neste estudo pacientes infectados pelo HIV sem uso de ARV.

É interessante observar que tanto a média da relação cintura/quadril quanto a média da espessura da gordura intra-abdominal dos nossos resultados foram próximas ou superiores aos pontos de corte de risco cardiovascular, independentemente do grupo em questão. A relação cintura/quadril foi de 0,94 $\pm 0,07$ em usuários de ARV e de 0,90 $\pm 0,07$ nos não usuários. O ponto de corte nas mulheres é de 0,8 e nos homens, de 0,95 (27). A espessura da gordura intraabdominal foi de $6,9 \pm 2,1 \mathrm{~cm}$ entre os usuários de ARV e de 6,0 $\pm 1,8 \mathrm{~cm}$ entre os $\operatorname{sem} \operatorname{ARV}(p=0,03)$ próximo ao corte de risco moderado estabelecido para o método e na população brasileira, de sete centímetros (28). Estas observações sugerem que pacientes não expostos a ARV também têm tendência a acúmulo de gordura central, e pacientes expostos a ARV apresentam um aumento da adiposidade central que poderia ser atribuído tanto à infecção pelo HIV per si quanto ao uso de anti-retrovirais, por ação direta e/ou pela melhora da resposta imunológica.

Alguns estudos prospectivos têm demonstrado incidência cumulativa elevada de novos casos de lipoatrofia e/ou lipo-hipertrofa após o início da terapia anti-retroviral (29-31). Estes estudos reforçam a hipótese de que as alterações morfológicas são associadas a ARV e são manifestações bastante comuns. Considerando-se que a distribuição regional da gordura, em particular o tecido gorduroso intra-abdominal, está associada com desordens metabólicas neuroendócrinas, principalmente resistência insulínica e síndrome metabólica, e com um grande aumento da morbidade e da mortalidade cardiovascular, parece razoável supor que os pacientes infectados pelo HIV em uso das drogas anti-retrovirais apresentem maior risco para doenças ateroscleróticas.
Os nossos dados demonstraram que pacientes em uso de ARV apresentam perfil lipídico mais aterogênico (aumento de triglicérides e de colesterol total) que os não usuários de $\mathrm{ARV}$, exceto em relação aos níveis de colesterol HDL.

A elevação de triglicerídeos é alteração bem descrita entre os infectados pelo HIV, inclusive antes da introdução da HAART (32). Vários estudos mostraram níveis de triglicérides maiores entre os infectados usuários de ARV quando comparados aos infectados não usuários de $\operatorname{ARV}(22,33,34)$ ou a grupo controle de não infectados $(17,35,36)$.

A elevação dos níveis de colesterol total também foi bem definida entre os usuários de ARV quando comparados com pacientes não usuários $(22,33,34,37)$ ou com grupo controle de não infectados $(17,37)$.

Os nossos dados de níveis mais elevados de colesterol HDL entre os usuários de ARV não estão de acordo com o observado em outros estudos. Alguns trabalhos mostraram níveis similares entre pacientes não tratados com $\mathrm{ARV}$ e os tratados com esquema incluindo inibidores de protease (IP) $(36,37)$. Outros autores encontraram níveis de colesterol HDL menores nos pacientes em uso de esquemas anti-retrovirais baseados em inibidores da transcriptase reversa análogos de nucleosídeos (ITRN) e IP $(21,22)$. Apenas Hadigan e cols. (38) encontraram resultados concordantes com os nossos, inclusive com concentrações médias de colesterol HDL ainda mais baixas. Todavia, este estudo selecionou pacientes infectados com relato de perda de peso ponderal significativa em relação ao início da infecção pelo HIV, fato que pode explicar baixos níveis de colesterol HDL. No nosso estudo, os pacientes infectados pelo HIV em sua maioria (71\%) tinham HDL abaixo do corte de risco $(40 \mathrm{mg} / \mathrm{dl}) \mathrm{e}$ apresentaram concentração média plasmática baixa (34 $\pm 12 \mathrm{mg} / \mathrm{dl}$ ), o que está de acordo com a literatura $(2,17,18,22,33)$. Desse modo, o que se pode apreender é que, no tocante ao colesterol HDL, os pacientes infectados pelo HIV em geral, usuários ou não de antiretrovirais, apresentam nível médio bastante baixo, o que sugere um maior risco cardiovascular independente do uso de anti-retrovirais.

Além disto, este perfil lipídico aterogênico de nossos pacientes está de acordo com o padrão de risco para doença metabólica e cardiovascular da distribuição de gordura corporal, com acúmulo central e perda periférica. Nosso estudo reforça o fato de que o uso de anti-retrovirais parece ser determinante para o surgimento de alterações lipídicas, seja por ação direta dos fármacos ou por modificações que possam provocar na relação hospedeiro-vírus. 
Os resultados deste estudo mostraram entre os pacientes em uso de ARV níveis mais elevados de glicemias aos 60 e aos 120 minutos após dextrosol e maior área sob a curva da variação glicêmica além de maiores valores de insulinemia de jejum e do índice HOMAIR quando comparados aos não usuários de ARV.

Em 1997, após a introdução da HAART, o FDA relatou a ocorrência de 83 casos de hiperglicemia em pacientes infectados pelo HIV e em uso de inibidores de protease, sugerindo associação entre transtornos da homeostase da glicose e estes medicamentos (39). Desde então, muitas hipóteses têm sido levantadas em relação às alterações glicêmicas. Sugerese, por exemplo, que o uso de inibidores de protease estaria associado a aumento de resistência insulínica sem, contudo, aumentar a prevalência de diabetes mellitus (40). Outra hipótese é de que a resistência insulínica seja conseqüente à própria infecção pelo HIV e não secundária ao uso de ARV (41). Nossos dados vão contra tal afirmativa, pois encontramos maior resistência insulínica entre os usuários de ARV.

Walli e cols. (34) também observaram maior resistência insulínica (definida pelo corte de dois desvios-padrão à resposta glicêmica ao teste de tolerância a insulina intravenosa), maiores concentrações de glicemia e insulinemia de jejum em pacientes usuários de ARV. Por sua vez, Saint-Marc e cols. (21, 22) encontraram valores semelhantes de glicemia de jejum e duas horas após sobrecarga oral e para insulinemia de jejum em usuários e não usuários de ARV, sendo que os pacientes em uso de esquema anti-retroviral com IP apresentaram níveis mais altos de peptídeo C.

Merece destaque o estudo de Mulligan e cols. (42), que demonstraram um aumento da insulinemia de jejum e de HOMA-IR após introdução de um IP ao esquema anti-retroviral, fato não observado após a introdução de um inibidor da transcriptase reversa não análogo de nucleosídeos (ITRNN).

Em conjunto, os nossos dados para o metabolismo da glicose sugerem que pacientes infectados em uso de ARV têm uma menor sensibilidade à insulina. Isto está de acordo com o já observado para as alterações lipídicas e, sobretudo, para as alterações da distribuição de gordura corporal.

Os benefícios da terapia anti-retroviral de alta potência na evolução da infecção pelo HIV estão bem estabelecidos e, com o aumento da sobrevida dos pacientes, as alterações tardias da infecção pelo HIV assumem um papel cada vez mais importante. Neste contexto, podemos destacar a síndrome de lipodistrofia do HIV. Indiscutivelmente, a infecção pelo HIV e o uso de HAART devem ser consideradas condições de risco para transtornos metabólicos e cardiovasculares, e coloca outros profissionais como endocrinologista, cardiologista e nutricionista na condução destes pacientes.

Ainda não existe consenso no que diz respeito à abordagem terapêutica da síndrome metabólica no HIV. O tratamento consiste no uso de medicamentos disponíveis para controle lipídico e glicêmico. Parecenos bastante razoável propor uma abordagem preventiva sistemática em todos os pacientes infectados pelo HIV, através de medidas para melhora da qualidade de vida, como interrupção do tabagismo, evitar excesso ponderal e sedentarismo, manter bons hábitos alimentares. Desta forma, busca-se uma redução no risco cardiovascular destes pacientes, até que seja melhor compreendida a fisiopatologia da síndrome e medidas terapêuticas mais eficazes possam ser estabelecidas.

\section{REFERÊNCIAS}

1. Miller KD, Jones E, Yanovski JA, Shankar R, Feurstein I, FalIoon J. Visceral abdominal-fat accumulation associated with use of indinavir. Lancet 1998;35:871-5.

2. Carr A, Samaras K, Burton S, Law M, Freund J, Chisholm DJ, et al. A syndrome of peripheral lipodystrophy, hiperlipidemia, insulin resistance in patients receiving HIV protease inhibitors. AIDS 1998;12:51-8.

3. Visnegarwala F, Krause KL, Musher DM. Severe diabetes associated with protease inhibitor therapy. Ann Int Med 1997;127:947.

4. Viraben $R$, Aquilina C. Indinavir associated lipodystrophy. AIDS 1998:12:37-9.

5. Montessori V, Press N, Harris M, Akagi L, Montaner JS. Adverse effects of antiretroviral therapy for HIV infection. CMAJ 2004; 170(2):229-38.

6. Grundy SM, Brewer HB, Cleeman JI, Smith SC, L'Enfant C. Definition of metabolic syndrome: report of the National Heart, Lung, and Blood Institute/American Heart Association conference on scientific issues related to definition. Circulation 2004; 109:433-8.

7. Hogg RS, O'Shaughnessy MV, Gataric N, Yip B, Craib K, Svhechter MT, et al. Decline in deaths from Aids due to new antiretrovirals (letter). Lancet 1997;349:1294.

8. Palella FJ Jr, Delaney KM, Moornan AC, Loveless MO, Fuhrer $J$, Satten GA, et al. Declining of morbidity and mortality among patients with advanced human immunodeficiency virus infection. N Eng J Med 1998;338:853-60.

9. Marins JRP, Jamal LF, Chen SY, Barros MB, Hudes ES, Barbosa $A A$, et al. Dramatic improvement in survival among adult Brazilian AIDS patients. AIDS 2003;17:1675-82.

10. Radominski RB, Hebroso DP, Cerri GG, Halpern A. O uso da ultra-sonografia na avaliação da distribuição de gordura abdominal. Arq Bras Endocrinol Metab 2000;44:5-12.

11. Durnin JVGA, Womersley J. Body fat assessed from total body density and its estimation from skinfold thickness: measurements on 481 men and women aged from 16-72 years. Brit J Nutr 1974;32:77-97.

12. Lukaski HC. Methods for the assessment of human body composition: traditional and new. Am J Clin Nutr 1987;46:537-56.

13. Armellini F, Zamboni M, Robbi R, Tudesco T, Rigo L, Bergamo-Andreis IA, et al. Total and intra abdominal fat measurements by ultrasound and computerized tomography. Inter J Obes 1983;17:209-14. 
14. Matthews DR, Hosker JP, Rudenski AS, Naylor BA, Treacher DF, Turner RC. Homeostasis model assessment: insulin resistance and $\beta$-cell function from fasting plasma glucose and insulin concentration in man. Diabetologia 1985;28:412-9.

15. MINISTÉRIO DA SAÚDE. Recomendações para terapia anti-retroviral em adultos e adolescentes infectados pelo HIV, 2001.

16. Heath KV, Hogg RS, Chan KJ, Harris M, Montessori V, O'Shaughnessy, et al. Lipodystrophy-associated morphological, cholesterol and triglyceride abnormalities in a population-based HIV-AIDS treatment database. AIDS 2001;15:231-9.

17. Carr A, Samaras S, Thorisdottir A, Kaufmann GR, Chisholm DJ, Cooper DA. Diagnosis, prediction and natural course of HIV-1 protease-inhibitor-associated lipodystrophy, hyperlipidaemia, and diabetes mellitus: a cohort study. Lancet 1999;353:2093-9.

18. Yanovski JA, Miller KD, Kino T, Friedman TC, Chrousos GP, Tsigos $\mathrm{C}$, et al. Endocrine and metabolic evaluation of human immunodeficiency virus-infected patients with evidence of protease inhibitor-associated lipodystrophy. J Clin Endocrinol Metab 1999;84:1925-31.

19. Kotler DP, Thea DM, Heo M, Allison, DB, Engelson ES, Wang $\mathrm{J}$, et al. Relative influences of sex, race, environment, and HIV infection on body composition in adults. Am J Clin Nutr 1999;69:432-9.

20. Kotler DP, Rosenbaum K, Wang J, Pierson RN. Studies of body composition and fat distribution in HIV-infected and control subjects. J Acquir Immune Defic Syndr 1999;20:228-37.

21. Saint-Marc T, Partisani M, Poizot-Martin I, Bruno F, Rouviere $\mathrm{O}$, Lang JM, et al. A syndrome of peripheral fat wasting (lipodystrophy) in patients receiving long-term nucleoside analogue therapy. AIDS 1999;13:1659-67.

22. Saint-Marc T, Partisani M, Poizot-Martin I, Rouviere O, Bruno $\mathrm{F}$, Avellaneda R, et al. Fat distribution evaluated by computed tomography and metabolic abnormalities in patients undergoing antiretroviral therapy: preliminary results of the LIPOCO study. AIDS 2000;14:37-49.

23. Deprés JP. Lipoprotein metabolism in abdominal obesity. Prog Obes Res J 1990;285-90.

24. Wannamethee SG, Shaper AG, Morris RW. Measures of adiposity in the identification of metabolic abnormalities in elderly men. Am J Clin Nutr 2005;81:1313-21.

25. Wei M, Gaskill SP, Haffner SM, Stern MP. Waist circumference as the best predictor of noninsulin dependent diabetes mellitus compared to body mass index, waist hip ratio and other anthropometric measurements in Mexican-Americans. Obes Res 1997;5:16-23.

26. Martínez E, Bianchi L, García-Viejo MA, Bru C, Gatell JM. Sonographic assessment of regional fat in HIV1-infected people. Lancet 2000;356:1412-3.

27. Lean MEJ, Han TS, Morrison CE. Waist circumference as a measure for indicating need for weight management. Brit Med J 1995;311:158-161.

28. Leite CC, Matsuda D, Wajchenberg BL, Cerri GG, Halpern A. Correlação da medida de espessura intra-abdominal medida pela ultra-sonografia com os fatores de risco cardiovascular. Arq Bras Endocrinol Metab 2000;44:49-56.

29. Tsiodras S, Mantzoros C, Hammer S, Samore M. Effects of protease inhibitors on hyperglycemia, hyperlipidemia and lipodystrophy - a 5-year cohort study. Arch Intern Med 2000;160(13):2050-6.
30. Heath K, Chan KJ, Singer J, O'Shaughnessey MV, Montanes JS, Hogg RS. Incidence of morphological and lipid abnormalities: Gender and treatment differentials after initiation of first antiretroviral therapy. Inter J Epidemiol 2002;31:1016-20.

31. Madge S, Kinloch-de-Loes S, Mercey D, Johnson MA, Weller IV. Lipodystrophy in patients naïve to HIV protease inhibitors (correspondence) AIDS 1999;13(6):735-7.

32. Grunfeld C, Feingold KR. Metabolic disturbances and wasting in the acquired immunodeficiency syndrome. $\mathbf{N}$ Eng $\mathbf{J}$ Med 1992;327:329-37.

33. Caramelli B, Bernoche CSM, Sartori MC, Sposito AC, Santos RD, Monachini MC, et al. Hiperlipidemia related to the use of HIV-protease inhibitors: natural history and results of treatment with fenofibrate. Braz J Infec Dis 2001;5:332-8.

34. Walli R, Herfort O, Michl GM, Demant T, Jäger H, Dieterle C, et al. Treatment with protease inhibitors associated with peripheral insulin resistance and impaired oral glucose tolerance in HIV-1-infected patients. AIDS 1998;12:F167-73.

35. Ledru E, Christeff N, Patey O, Truchis P, Melchior JC, Gougeon ML. Alteration of tumor necrosis factor- $\alpha$ T-cell homeostasis following potent antiretroviral therapy; contribution to the development of human immunodeciciency virus-associated lipodystrophy syndrome. Blood 2000; 95:3191-8.

36. Depairon M, Chessex S, Sudre P, Rodondi N, Doser N, Chave $\mathrm{JP}$, et al. Premature atherosclerosis in HIV-infected individuals-focus on protease inhibitor therapy. AIDS 2001;15:32934.

37. Berthold HK, Parhofer KG, Ritter MM, Addo M, Wasmuth JC, Schliefer K, et al. Influence of protease inhibitor therapy on lipoprotein metabolism. J Inter Med 1999;246:567-75.

38. Hadigan C, Corcovan C, Stanley T, Piecuch S, Klibanski A, Grinspoon S. Fasting hiperinsulinemia in human immunodeficiency virus-infected men: relationship to body composition, gonadal function, and protease inhibitor use. J Clin Endocrinol Metab 2000;85:35-41.

39. Lumpkin M. FDA Public Health Advisory: Reports of diabetes and hyperglycemia in patients receiving proteae inhibitors for the treatment of human immunodeficiency virus (HIV). Washington, DC: US Government Printing Office, 1997.

40. Lee ECC, Walmsley S, Fantus IG. New onset diabetes mellitus associated with protease inhibitors therapy in an HIV-positive patient: case report and review. CMAF 1999;161:161-4.

41. Dube MP. Disorders of glucose metabolism in patients infected with human immunodeficiency virus. Clin Infect Dis 2000:31:1467-75.

42. Mulligan K, Grunfeld DC, Tai VW, Algren H, Pang M, Chernoff $\mathrm{DN}$, et al. Hyperlipidemia and insulin resistance are induced by protease inhibitors independent of changes in body composition in patients with HIV infection. J Acquir Immune Defic Syndr 2000;23:3543.

\section{Endereço para correspondência:}

Milena Maria Moreira Guimarães

Rua Hermílio Alves 159 / 404

31010-070 Belo Horizonte, MG

E-mail: milenamg@uol.com.br 